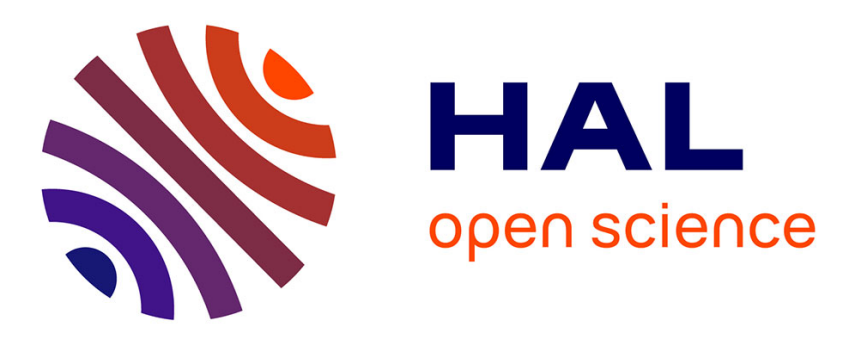

\title{
An Analytical Fiber ODF Reconstruction in 3D Polarized Light Imaging
}

\author{
Abib Alimi, Yves Usson, Pierre-Simon Jouk, Gabrielle Michalowicz, Rachid \\ Deriche
}

\section{- To cite this version:}

Abib Alimi, Yves Usson, Pierre-Simon Jouk, Gabrielle Michalowicz, Rachid Deriche. An Analytical Fiber ODF Reconstruction in 3D Polarized Light Imaging. ISBI 2018 - IEEE International Symposium on Biomedical Imaging, Apr 2018, Washington, D.C., United States. pp.1-4. hal-01688789

\section{HAL Id: hal-01688789 \\ https://hal.inria.fr/hal-01688789}

Submitted on 19 Jan 2018

HAL is a multi-disciplinary open access archive for the deposit and dissemination of scientific research documents, whether they are published or not. The documents may come from teaching and research institutions in France or abroad, or from public or private research centers.
L'archive ouverte pluridisciplinaire $\mathbf{H A L}$, est destinée au dépôt et à la diffusion de documents scientifiques de niveau recherche, publiés ou non, émanant des établissements d'enseignement et de recherche français ou étrangers, des laboratoires publics ou privés. 


\title{
An Analytical Fiber ODF Reconstruction in 3D Polarized Light Imaging
}

\author{
Abib Alimi ${ }^{\dagger}$, Yves Ussou ${ }^{\star}$, Pierre-Simon Jouk ${ }^{\star}$, Gabrielle Michalowicz ${ }^{\star}$ and Rachid Deriche ${ }^{\dagger \S}$ \\ †Université Côte d'Azur, Inria, France \\ * TIMC-IMAG, Université Grenoble Alpes, Grenoble, F-38000, France
}

\begin{abstract}
Three dimensional polarized light imaging (3D-PLI) utilizes the birefringence in postmortem tissue to map its spatial fiber structure at a submillimeter resolution. We propose an analytical method to compute the fiber orientation distribution function (ODF) from high-resolution vector data provided by 3D-PLI. This strategy enables the bridging of high resolution 3D-PLI to diffusion magnetic resonance imaging with relatively low spatial resolution. First, the fiber ODF is modeled as a sum of $K$ orientations on the unit sphere and expanded with a high order spherical harmonics series. Then, the coefficients of the spherical harmonics are derived directly with the spherical Fourier transform. We quantitatively validate the accuracy of the reconstruction against synthetic data and show that we can recover complex fiber configurations in the human heart at different scales.
\end{abstract}

Index Terms - fiber orientation distribution function ODF, 3D-PLI, polarized light imaging, spherical harmonics, spherical Fourier transform

\section{INTRODUCTION}

Diffusion magnetic resonance imaging (dMRI) is the only imaging technique able to assess the structural architecture of human fibrous organs, in vivo and non invasively. However, different imaging methods at multiple scales are necessary to improve our understanding of brain and heart disorders. By providing microscopic fiber orientation measurements, 3D-PLI is not only a potential technique to validate diffusion magnetic resonance imaging results but also a complementary imaging approach. Therefore, it is compulsory to bridge the spatial scale information from micro to millimeter dimensions. The orientation distribution function, which is widely used in dMRI community [1, 2], is a perfect tool to compare dMRI and 3D-PLI fiber orientations estimates.

To this end, Axer et al. [3] introduced an estimate of fiber ODF derived from 3D-PLI high-resolution vector data, which they called pliODF. Starting from the high-resolution fiber orientation map (FOM) [4] illustrated in Figure 1], their approach is implemented as follows: 1. Define super-voxels to downsample the high-resolution data measured from histological tissue sections. Super-voxels are rectangular compartments in one or a series of FOMs. In a super-voxel, there are $K=$ rows $\times$ columns $\times$ slices high-resolution orientations. 2. Create a normalized directional histogram on the unit sphere from discretized distribution of fiber orientation vectors in each super-voxel. 3. The spherical harmonics (SH) series are used to approximate the discretized directional histogram within each super-voxel. This is the first attempt to estimate the fiber ODF from the high-resolution 3D-PLI data and it benefits from the important concept of super-voxel which allows to estimate the fiber ODF at different spatial scales. However, this technique has two main limitations. First, it introduces angular errors in the recovered pliODF due to the discretization of the directional histogram, which is defined empirically and limits the order of the spherical harmonics expansion. Second, it has no regularization process and the least square solution approximates a discrete set of the SH coefficients.

In this work, we overcome these limitations by proposing an analytical procedure to derive the fiber ODF in order to assess the spatial distribution of fiber orientations within a super-voxel. Our main contribution is a new approach to generate fiber ODF within a super-voxel of high-resolution 3D-PLI vector data by:

1. modeling the fiber ODF as a sum of $K$ Dirac delta functions

2. and deriving its spherical harmonics coefficients using the spherical Fourier transform.

This serves as a important step towards our major goal which is the validation of diffusion MRI results such as tractography via 3D-PLI. More specifically, this work leads to the comparison of 3D fiber organizations resulting from different imaging modalities and at different scales through the fiber orientation distribution functions.

The rest of the paper continues as follows: our reconstruction method of the fiber ODF is described in section 2, then it is evaluated on both synthetic and human datasets in section 3.

\footnotetext{
$\S$ This work was supported by ANR "MOSIFAH" under ANR-13MONU-0009-01.
} 


\section{FIBER ORIENTATION DISTRIBUTION FUNCTIONS RECONSTRUCTION}

As a function defined on the unit sphere $\mathbb{S}^{2}$, the fiber ODF can be expanded as linear combination of spherical harmonics. Here we give a brief background information on spherical harmonics before presenting how we obtain the exact SH coefficients that uniquely characterize our 3D-PLI-based fiber ODF.

\subsection{Spherical harmonics}

The spherical harmonics (SH) of order $l$ and phase factor $m$ are defined as

$$
Y_{l}^{m}(\theta, \phi)=N_{l}^{m} P_{l}^{m}(\cos \theta) e^{j m \phi}
$$

where $N_{l}^{m}$ is a normalization coefficient, $P_{l}^{m}$ are the associated Legendre polynomials, $\theta \in[0, \pi]$ and $\phi \in[0,2 \pi)$ are the colatitude and azimuth, respectively. The SH $\left\{Y_{l}^{m}:-l \leqslant\right.$ $m \leqslant l, l=0,1, \ldots\}$ form an orthonormal basis over $L^{2}\left(\mathbb{S}^{2}\right)$ and any square integrable function $f(\theta, \phi) \in L^{2}\left(\mathbb{S}^{2}\right)$ can be expressed as

$$
f(\theta, \phi)=\sum_{l=0}^{\infty} \sum_{m=-l}^{l} c_{l m} Y_{l}^{m}(\theta, \phi)
$$

where $c_{l m}$ are the SH coefficients of $f$. These coefficients are obtained by computing the spherical Fourier transform of $f$ defined as

$$
c_{l m}=\int_{\mathbb{S}^{2}} f(\mathbf{w}) \overline{Y_{l}^{m}}(\mathbf{w}) d \mathbf{w}
$$

for $\mathbf{w} \in \mathbb{S}^{2}$ and the overbar denotes the conjugation. The unit vector $\mathbf{w}$ is related to $(\theta, \phi)$ by

$$
\mathbf{w}(\theta, \phi)=\left[\begin{array}{lll}
\sin \theta \cos \phi & \sin \theta \sin \phi & \cos \theta
\end{array}\right]^{t}
$$

where ${ }^{t}$ indicates transposition. In this paper, both functions $f(\theta, \phi)$ and $f(\mathbf{w})$ are assumed to be equal and written interchangeably to simplify notations.

\section{2. pliODF}

As aforementioned, [3] estimate the fiber ODF in a given super-voxel using SH expansion to approximate the normalized directional histogram. The SH coefficients are determined with a linear least square method. This problem can be expressed in matrix format as $H=B C$, with $H$ the vector entries of the histogram, $B$ is the basis matrix of the truncated $\mathrm{SH}$ of maximum order or bandlimit $L_{\max }$ and $C$ is the $\mathrm{SH}$ coefficients vector whose length increases non-linearly with $L_{\max }$ [2, 3]. The least square solution for $C$ gives an approximation of a discrete set of the SH coefficients because of the discretization of the directional histogram, which limits the $L_{\max }$ of the $\mathrm{SH}$ and the angular resolution of the recovered fiber ODF.

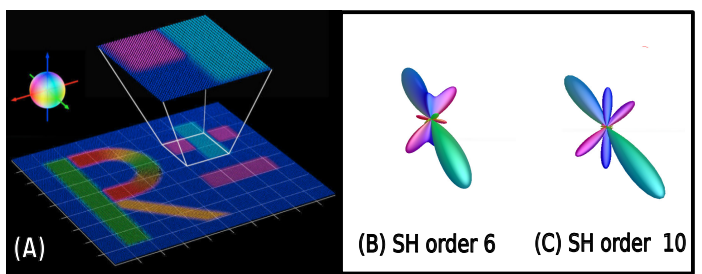

Fig. 1. Fiber ODF generation. (A) Simulated dataset shows the principle of super-voxel definition, adapted from [3]. The corresponding fiber ODF is reconstructed in (B) with pliODF technique while in (C) our approach improves the angular resolution.

In a previous work [5], we improved this solution by increasing $L_{\max }$ and regularizing the estimated SH coefficients using the Laplace-Beltrami operator. The Laplace-Beltrami operator $\Delta_{b}$ is well-known in the dMRI community [2] and satisfies the relation $\Delta_{b} Y_{l}^{m}=-l(l+1) Y_{l}^{m}$. Therefore, the solution of the regularized SH coefficients writes $C=$ $\left(B^{T} B+\lambda L\right)^{-1} B^{T} H$ where $\lambda$ is the regularization weight and $L$ denotes the regularization matrix with entries $l^{2}(l+1)^{2}$ along the diagonal.

Although [5] improve the angular resolution of the estimated pliODF, this solution still provides a discrete set of the SH coefficients due to the discretization of the direction histogram. We therefore propose, in the next section, to determine all the exact coefficients of the SH expansion of our new model of fiber ODF.

\subsection{Our fiber ODF as sum of $K$ Diracs}

In each voxel of the FOM, we propose to describe the unit fiber orientation vector as a two dimensional Dirac delta function $\delta$ on the sphere. Therefore, in a super-voxel containing $K$ orientations, the fiber orientation distribution function $f$ can be modeled as the sum of $K$ Diracs [6], that is

$$
f(\theta, \phi)=\frac{1}{K} \sum_{k=1}^{K} \delta\left(\cos \theta-\cos \theta_{k}\right) \delta\left(\phi-\phi_{k}\right)
$$

Note that $f(\theta, \phi)$ is completely defined by parameters $\left(\theta_{k}, \phi_{k}\right)$ of the $K$ orientations located in the super-voxel. In the next step, the coefficients of the SH expansion of $f$ are determined.

\subsection{Computation of the SH coefficients}

Unlike [3] who used a linear least square method to get the coefficients of the spherical harmonics expansion, we directly recover the coefficients that uniquely define our fiber ODF $f$ by computing the spherical Fourier transform through Eq. (3) since we know the parameters $\left(\theta_{k}, \phi_{k}\right)$ of all fiber orientation vectors determined from 3D-PLI analysis [7, 4], in a given super-voxel.

In Eq. 3), when $f$ is replaced by the collection of Diracs as defined from Eq. (5), the sifting property of the Diracs is 


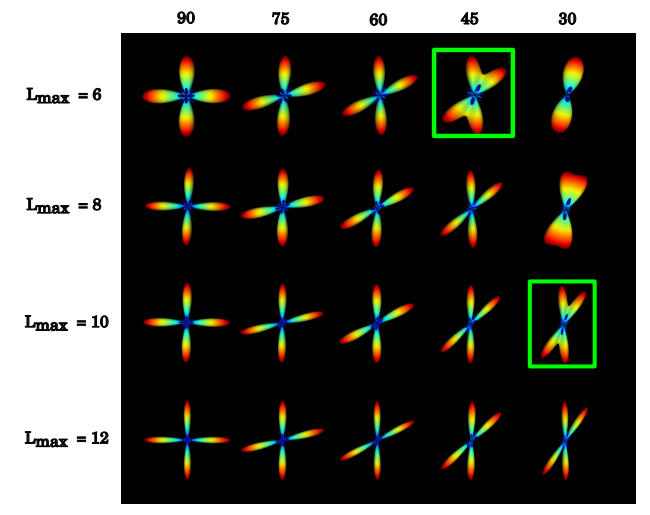

Fig. 2. Angular separation: $L_{\max }=10$ is enough to detect crossing fiber populations at acute angle $\alpha=30^{\circ}$.

applied, and the definition of the spherical harmonics in Eq. (1) is substituted, then the SH coefficients are given by

$$
c_{l m}=\frac{N_{l}^{m}}{K} \sum_{k=1}^{K} P_{l}^{m}\left(\cos \theta_{k}\right) e^{-j m \phi_{k}} .
$$

This solution for the $c_{l m}$ is the continuous equivalent of the discrete one approximated for pliODF. Eq. 6 is exact and a more accurate solution because it requires no discretization since all the high resolution fiber orientation vectors are taken into account in the computation. Furthermore, it can be evaluated at any $L_{\max }$.

We can now appraise the performance of our recovered fiber ODF in the following section.

\section{EVALUATING THE ODF}

\subsection{Synthetic data}

The synthetic data consists of a FOM divided into 5 supervoxels of size $K=10 \times 10 \times 1$ containing 100 orientations $\left(\theta_{k}, \phi_{k}\right)$ each, describing 2 fiber populations crossing each other at angles $\alpha=30^{\circ}, 45^{\circ}, 60^{\circ}, 75^{\circ}$ and $90^{\circ}$ respectively. We run our algorithm with $L_{\max }=6,8,10$ and 12 , we recall that $L_{\max }$ is the band-limit of the truncated SH expansion. Although our method is independent of the SH basis used, the real and symmetric basis defined in [2] is considered in this section.

Angular separation. We start by demonstrating the ability of our recovered ODF to resolve crossing fiber populations at different angles. Figure 2 shows that, with low $L_{\max }=6$, we are already able to separate fibers crossing at acute $\alpha=$ $45^{\circ}$. Furthermore, $L_{\max }=10$ is enough to detect and separate crossings at $\alpha=30^{\circ}$. Overall, our algorithm is able to find all crossing configurations at different angles except for $\alpha \leqslant 30^{\circ}$ when $L_{\max } \leqslant 8$. This result highlights that our method can improve angular resolution of the reconstructed fiber ODF in each super-voxel.

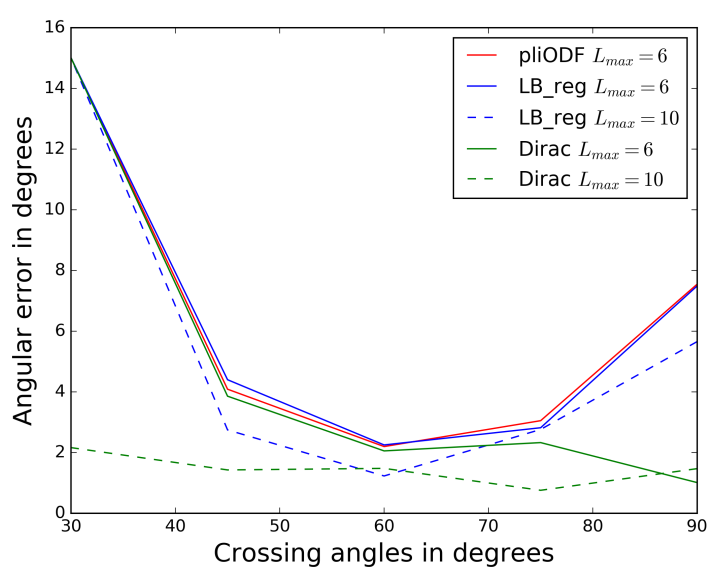

Fig. 3. Angular error: with $L_{\max }=10$ our method gives smallest error.

Angular error. For quantitative validation, we evaluate our algorithm using the angular error expressed as

$$
\beta=\frac{1}{K} \sum_{k=1}^{K} \arccos \left(\mathbf{v}_{k}^{t} \mathbf{w}_{k}\right)
$$

which is the distance between $\mathbf{v}_{k}$ the $k^{t h}$ ground truth orientation and $\mathbf{w}_{k}$ the corresponding recovered orientation in a super-voxel. Both orientations are defined as in Eq. 4 Figure 3 displays the angular errors in degrees obtained after ODF reconstruction with our algorithm noted Dirac $L_{\max }=\{6,10\}$ compared to pliODF at $L_{\max }=6$ as suggested by the authors in [3] and its regularized version LB reg at $L_{\max }=\{6,10\}$. Here, the angular error is plotted against crossing angles $\alpha$. Results indicate that our algorithm produces less error than the pliODF and LB_reg even at $L_{\max }=6$. At $L_{\max }=10$, we outperform LB_reg expect at $\alpha=60^{\circ}$ while pliODF, not plotted here, produces errors around $\beta=50^{\circ}$. Moreover, at $\alpha=30^{\circ}$ while the others give errors around $\beta=15^{\circ}$, our fiber ODF at $L_{\max }=10$ gives again the lowest error about $\beta=2^{\circ}$. It should be noted that this error is caused by the limited angular resolution of the selected $L_{\max }$ and not by errors introduced by our method. Indeed, this error decreases very significantly with high SH orders in all crossing configurations when using our algorithm. These results agree with our observations in the previous simulation.

\subsection{Human heart data}

We now perform our fiber ODF reconstruction approach on post-mortem human heart data from a healthy adult. The organ was frozen, sliced and imaged with respect to the PLI protocol in [7]. Figure 4(A) presents a coronal slice showing the left and the right ventricles. The imaging procedure, which does not require any staining of the tissue [7, 4], produced high-resolution data of $440 \times 620$ voxels in each FOM. 


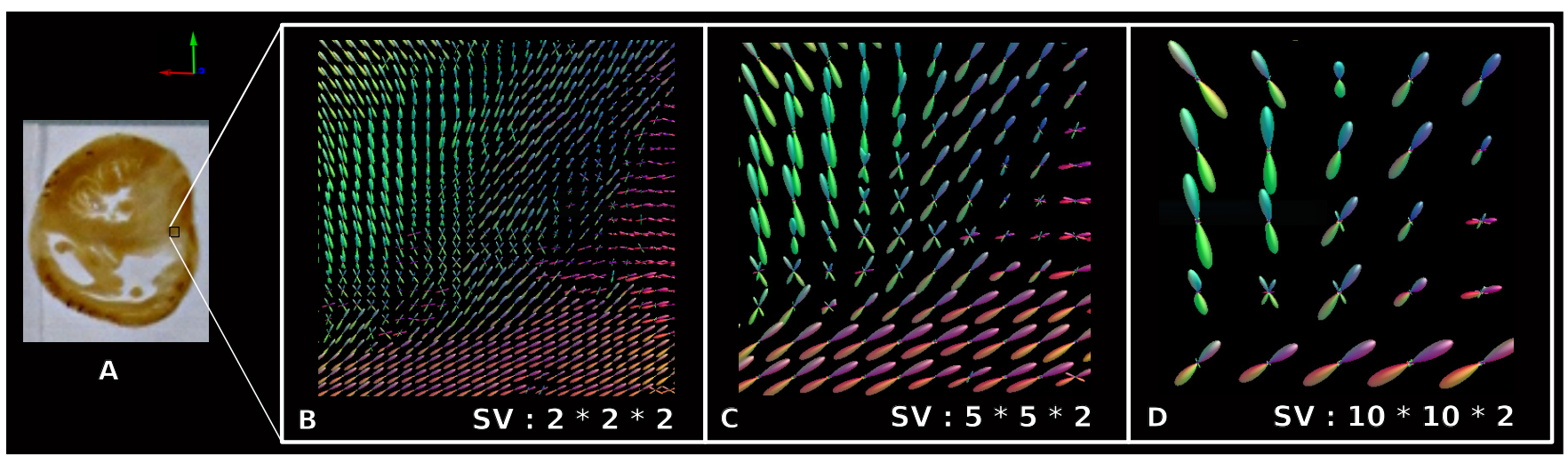

Fig. 4. Fiber ODFs reconstructed from coronal ventricular tissue slices at super-voxels sizes SV $2 \times 2 \times 2$ (B), $5 \times 5 \times 2(\mathbf{C})$ and $10 \times 10 \times 2(\mathbf{D})$. Each glyph represents the probability distribution of orientations over $\mathbb{S}^{2}$ and color-coded in RGB space.

Fiber ODFs are computed at different resolutions defined by the super-voxel size and Figure 4 (B), (C) and (D) display the results for super-voxels of size $2 \times 2 \times 2,5 \times 5 \times 2$ and $10 \times 10 \times 2$, respectively, where two consecutive and registered FOM slices were taken into account. Figure 4 underlines two important results in a zoomed region of the junction between the left and the right ventricles in (A). First, crossing fiber bundles are recovered in this intersection displaying a very complex structure with mixing orientations that can change very suddenly. Second, data can be integrated at different scales as (B), (C) and (D) show the same region at different spatial resolutions. This can be observed thanks to the downsampling of the high-resolution data into super-voxels.

\section{DISCUSSION AND CONCLUSION}

We proposed a new method to define and reconstruct the fiber ODF from high-resolution 3D-PLI datasets. To this end, the fiber ODF is modeled as a sum of $K$ 2D Diracs on the sphere and the coefficients of its $\mathrm{SH}$ expansion are calculated using the spherical Fourier transform. This constitutes an elegant solution for fiber ODF reconstruction from 3D-PLI data compared to the pliODF [3] which is limited by the arbitrary discretization of the directional histogram.

Evaluations on synthetic datasets show that our method outperforms the pliODF as the angular resolution of recovered fiber ODFs are considerably improved and their angular error is very low at high $\mathrm{SH} L_{\max }$. This is very important because introducing errors in the fiber ODFs limits the use of 3D-PLI as a gold standard. This angular error can be ameliorated since it depends on the technique used to find the peaks of the ODF. Results from human data agree with the high complex arrangement of the 3D myocytes in the junction between left and right ventricles as observed in [8] using $\mathrm{X}$-ray phase-contrast micro-tomography.

Finally our new approach to reconstruct the fiber orientation distribution function opens the door to aligning highresolution 3D-PLI data to dMRI data. Therefore, multimodal data analysis at voxel level will be investigated in our future works.

\section{REFERENCES}

[1] Tuch, "Q-ball imaging," Magnetic resonance in medicine, vol. 52, no. 6, pp. 1358-1372, 2004.

[2] Descoteaux et al, "Regularized, fast, and robust analytical Q-ball imaging," Magnetic resonance in medicine, vol. 58, no. 3, pp. 497-510, 2007.

[3] Axer et al, "Estimating fiber orientation distribution functions in 3D-polarized light imaging," Frontiers in neuroanatomy, vol. 10, 2016.

[4] Axer et al, "A novel approach to the human connectome: ultra-high resolution mapping of fiber tracts in the brain," Neuroimage, vol. 54, no. 2, pp. 1091-1101, 2011.

[5] Alimi et al, "Regularizing the ODF estimate with the Laplace-Beltrami operator in 3D Polarized Light Imaging," CoBCoM 2017 - Computational Brain Connectivity Mapping Winter School Workshop, Nov. 2017.

[6] Deslauriers-Gauthier et al, "The application of a new sampling theorem for non-bandlimited signals on the sphere: Improving the recovery of crossing fibers for low b-value acquisitions," Medical image analysis, vol. 30, pp. 46-59, 2016.

[7] Jouk et al, "Three-dimensional cartography of the pattern of the myofibres in the second trimester fetal human heart," Anatomy and embryology, vol. 202, no. 2, pp. 103-118, 2000.

[8] Varray et al, "Extraction of the 3D local orientation of myocytes in human cardiac tissue using X-ray phase-contrast micro-tomography and multi-scale analysis," Medical image analysis, vol. 38, pp. 117-132, 2017. 